\title{
Fixed point theorems for rational type $(\alpha-\Theta)$-contractions in controlled metric spaces
}

\author{
Jamshaid Ahmad ${ }^{\mathrm{a}}$, Durdana Lateef ${ }^{\mathrm{b}, *}$ \\ ${ }^{a}$ Department of Mathematics, University of Jeddah, P. O. Box 80327, Jeddah 21589, Saudi Arabia. \\ ${ }^{b}$ Department of Mathematics, College of Science, Taibah University, Al Madina Al Munawara, 41411, Kingdom of Saudi Arabia.
}

\begin{abstract}
This paper aims to define rational type $(\alpha-\Theta)$-contraction in controlled metric space and obtain some advanced fixed point theorems. The outcomes generalize and extend various famous results in the literature. An example and certain consequences are presented to illustrate the significance of established results.
\end{abstract}

Keywords: Fixed point, rational type $(\alpha-\Theta)$-contraction, controlled metric spaces.

2010 MSC: 54H25, 47H10.

(C)2020 All rights reserved.

\section{Introduction}

In 1906 Frechet provided the conception of metric space which was an axiomatic development in functional analysis. Due to its simplicity, it has been generalized by several researchers[1-19] in the recent past.

Czerwik [9] defined the conception of b-metric space in this way.

Definition 1.1 ([9]). Let $\mathcal{W} \neq \emptyset$ and $b \geqslant 1$. A mapping $\kappa_{b}: \mathcal{W} \times \mathcal{W} \rightarrow[0, \infty)$ is called a $b$-metric if these assertions hold:

(b1) $\kappa_{\mathrm{b}}(\mathrm{l}, \mathrm{s})=0 \Longleftrightarrow \mathrm{\imath}=\mathrm{s}$;

(b2) $\kappa_{b}(l, s)=\kappa_{b}(s, l)$ for all $\iota, s \in \mathcal{W}$;

(b3) $\kappa_{b}(l, z) \leqslant b\left[\kappa_{b}(l, s)+\kappa_{b}(s, z)\right]$ for all $\mathrm{l}, s, z \in \mathcal{W}$.

Then $\left(\mathcal{W}, \kappa_{b}\right)$ is called a b-metric space $(b-M S)$.

Kamran et al. [14] gave the notion of extended b-metric spaces in 2017.

Definition 1.2. Let $\mathcal{W} \neq \emptyset, p: \mathcal{W} \times \mathcal{W} \rightarrow[1, \infty)$ and $\kappa_{e}: \mathcal{W} \times \mathcal{W} \rightarrow[0, \infty)$. Then $\kappa_{e}$ is called an extended b-metric if

\footnotetext{
*Corresponding author

Email addresses: jkhan@uj .edu.sa (Jamshaid Ahmad), drdurdanamaths@gmail.com (Durdana Lateef)

doi: $10.22436 /$ jnsa.013.03.05
}

Received: 2019-04-17 Revised: 2019-05-18 Accepted: 2019-07-02 
(i) $\kappa_{e}(l, s)=0$ if and only if $\iota=s$;

(ii) $K_{e}(l, s)=K_{e}(s, l)$;

(iii) $\kappa_{e}(l, s) \leqslant p(l, s)\left[\kappa_{e}(l, z)+\kappa_{e}(s, z)\right]$

for all $\iota, s, z \in \mathcal{W}$. Then $\left(\mathcal{W}, \kappa_{e}\right)$ is said to be an extended b-metric space (Eb-MS).

Currently, a new type of a space was given by Mlaiki et al. [16].

Definition 1.3. Let $\mathcal{W} \neq \emptyset, p: \mathcal{W} \times \mathcal{W} \rightarrow[1, \infty)$, and $\kappa_{p}: \mathcal{W} \times \mathcal{W} \rightarrow[0, \infty)$. Then $\kappa_{p}$ is said to be a controlled metric if

(i) $\kappa_{p}(\iota, s)=0$ if and only if $\iota=s$;

(ii) $\kappa_{p}(l, s)=\kappa_{p}(s, l)$;

(iii) $\kappa_{p}(l, s) \leqslant p(l, z) \kappa_{p}(l, z)+p(s, z) \kappa_{p}(s, z)$

for all $\iota, s, z \in \mathcal{W}$. The pair $\left(\mathcal{W},\left(\kappa_{p}\right)\right.$ is called a controlled metric space (CMS).

In 2015, Samet et al. [13] gave the notion of $\Theta$-contraction in this way.

Definition 1.4. Let $\Theta:(0, \infty) \rightarrow(1, \infty)$ be a function satisfying:

$\left(\Theta_{1}\right) \Theta$ is nondecreasing;

$\left(\Theta_{2}\right)$ for each sequence $\left\{\iota_{n}\right\} \subseteq \iota^{+}, \lim _{n \rightarrow \infty} \Theta\left(\iota_{n}\right)=1 \Leftrightarrow \lim _{n \rightarrow \infty}\left(\iota_{n}\right)=0$;

$\left(\Theta_{3}\right) \exists 0<\mathrm{h}<1$ and $\vartheta \in(0, \infty]$ such that $\lim _{\mathfrak{l} \rightarrow 0^{+}} \frac{\Theta(\mathfrak{l})-1}{\mathrm{l}^{\mathrm{h}}}=\vartheta$.

A self mapping $\mathcal{O}: \mathcal{W} \rightarrow \mathcal{W}$ is called $\Theta$-contraction if $\exists \Theta$ satisfying $\left(\Theta_{1}\right)-\left(\Theta_{3}\right)$ and $k \in(0,1)$ such that

$$
\kappa(\mathcal{O}, \mathcal{O} s) \neq 0 \Longrightarrow \Theta(\kappa(\mathcal{O}, \mathcal{O} s)) \leqslant[\Theta(\kappa(\iota, s))]^{k}
$$

for all $\iota, s \in \mathcal{W}$.

Theorem 1.5 ([13]). If $\mathcal{O}: \mathcal{W} \rightarrow \mathcal{W}$ be a $\Theta$-contraction on complete metric space $(\mathcal{W}, \mathrm{\kappa})$, then $\exists \iota^{*} \in \mathcal{W}$ such that $\iota^{*}=\mathcal{O} \iota^{*}$.

We represent by the $\Omega$, the family of all above mapping $\Theta$ satisfying the above assertions $\left(\Theta_{1}\right)-\left(\Theta_{3}\right)$ to be consistent with Samet et al. [13].

In this paper, we define rational type $(\alpha, \Theta)$-contraction in the setting of complete CMS to obtain some generalized results.

\section{Main results}

Definition 2.1. Let $\left(\mathcal{W}, \kappa_{p}\right)$ be a CMS. A function $\mathcal{O}: \mathcal{W} \rightarrow \mathcal{W}$ is said to be a rational type $(\alpha, \Theta)$-contraction if $\exists \alpha: \mathcal{W} \times \mathcal{W} \rightarrow \mathbb{R}^{+}, k \in(0,1)$ and $\Theta \in \Omega$ such that

$$
\alpha(\iota, s) \Theta\left(\kappa_{p}(\mathcal{O},, \mathcal{O} s)\right) \leqslant \Theta(M(l, s))^{k},
$$

where

$$
M(\iota, s)=\max \left\{\kappa_{p}(\iota, s), \kappa_{p}(\iota, \mathcal{O} \iota), \kappa_{p}(s, \mathcal{O} s), \frac{\kappa_{p}(\iota, \mathcal{O} \iota) \kappa_{p}(s, \mathcal{O} s)}{1+\kappa_{p}(\iota, s)}\right\},
$$

$\forall \mathrm{l}, \mathrm{s} \in \mathcal{W}$ with $\kappa_{\mathrm{p}}(\mathcal{O} \mathrm{l}, \mathcal{O} \mathrm{s})>0$.

From now onward, we consider $\left(\mathcal{W}, \kappa_{p}\right)$ as complete controlled metric space.

Theorem 2.2. Let $\left(\mathcal{W}, \kappa_{\mathrm{p}}\right)$ is a complete $C M S$ and $\mathcal{O}: \mathcal{W} \rightarrow \mathcal{W}$ be a rational type $(\alpha, \Theta)$-contraction such that:

(i) $\mathcal{O}$ is $\alpha$-admissible;

(ii) $\exists \iota_{0} \in \mathcal{W}$ such that $\alpha\left(\iota_{0}, \mathcal{O} \iota_{0}\right) \geqslant 1$; 
(iii) $\mathcal{O}$ is continuous;

(iv) $\sup _{m \geqslant 1} \lim _{i \rightarrow \infty} \frac{p\left(\mathfrak{t}_{i+1}, \mathfrak{l}_{i+2}\right) p\left(\mathfrak{L}_{i+1}, \iota_{m}\right)}{p\left(\iota_{i}, \iota_{i+1}\right)}<1$.

In addition, assume that, for every $\iota \in \mathcal{W}$, we have $\lim _{n \rightarrow \infty} p\left(\iota_{n}, \iota\right)$ and $\lim _{n \rightarrow \infty} p\left(\iota, \iota_{n}\right)$ exist and are finite. Then, $\exists \iota^{*} \in \mathcal{W}$ such that $\iota^{*}=\mathcal{O} \iota^{*}$.

Proof. Suppose $\mathfrak{\iota}_{0} \in \mathcal{W}$ is such that $\alpha\left(\iota_{0}, \mathcal{O} \iota_{0}\right) \geqslant 1$. We generate $\left\{\iota_{n}\right\}$ in $\mathcal{W}$ by $\iota_{n+1}=\mathcal{O} \iota_{n}, \forall n \in \mathbb{N}$. Obviously, if $\exists n_{0} \in \mathbb{N}$ for which $\iota_{n_{0}+1}=\mathfrak{l}_{n_{0}}$, then $\mathcal{O} \mathfrak{l}_{n_{0}}=\mathfrak{l}_{n_{0}}$ and the proof is finished. Thus, we assume that $\iota_{n+1} \neq \iota_{n}, \forall n \in \mathbb{N}$. By using (i) and (ii), it is obvious that

$$
\alpha\left(\iota_{n}, \iota_{n+1}\right) \geqslant 1,
$$

$\forall n \in \mathbb{N}$. Thus by (2.1), we obtain

$$
1<\Theta\left(\kappa_{p}\left(\iota_{n}, \iota_{n+1}\right)\right)=\Theta\left(\kappa_{p}\left(\mathcal{O} \iota_{n-1}, \mathcal{O} \iota_{n}\right)\right) \leqslant \alpha\left(\iota_{n}, \iota_{n+1}\right) \Theta\left(\kappa_{p}\left(\mathcal{O} \iota_{n-1}, \mathcal{O} \iota_{n}\right)\right)
$$

Since $\mathcal{O}$ is a rational type $(\alpha, \Theta)$-contraction, so $\forall n \in \mathbb{N}$, we can write

$$
\begin{aligned}
1 & <\Theta\left(\kappa_{p}\left(\iota_{n}, \iota_{n+1}\right)\right) \leqslant \alpha\left(\iota_{n}, \iota_{n+1}\right) \Theta\left(\kappa_{p}\left(\mathcal{O} \iota_{n-1}, \mathcal{O} \iota_{n}\right)\right) \\
& \leqslant \Theta\left(M\left(\iota_{n-1}, \iota_{n}\right)\right)^{k} \\
& =\Theta\left(\max \left\{\kappa_{p}\left(\iota_{n-1}, \iota_{n}\right), \kappa_{p}\left(\iota_{n-1}, \mathcal{O} \iota_{n-1}\right), \kappa_{p}\left(\iota_{n}, \mathcal{O} \iota_{n}\right), \frac{\kappa_{p}\left(\iota_{n-1}, \mathcal{O} \iota_{n-1}\right) \kappa_{p}\left(\iota_{n}, \mathcal{O} \iota_{n}\right)}{1+\kappa_{p}\left(\iota_{n-1}, \iota_{n}\right)}\right\}\right)^{k} \\
& =\Theta\left(\max \left\{\kappa_{p}\left(\iota_{n-1}, \iota_{n}\right), \kappa_{p}\left(\iota_{n-1}, \iota_{n}\right), \kappa_{p}\left(\iota_{n}, \iota_{n+1}\right), \frac{\kappa_{p}\left(\iota_{n-1}, \iota_{n}\right) \kappa_{p}\left(\iota_{n}, \iota_{n+1}\right)}{1+\kappa_{p}\left(\iota_{n-1}, \iota_{n}\right)}\right\}\right)^{k} \\
& \leqslant \Theta\left(\max \left\{\kappa_{p}\left(\iota_{n-1}, \iota_{n}\right), \kappa_{p}\left(\iota_{n-1}, \iota_{n}\right), \kappa_{p}\left(\iota_{n}, \iota_{n+1}\right), \kappa_{p}\left(\iota_{n}, \iota_{n+1}\right)\right\}\right)^{k} \\
& =\Theta\left(\max \left\{\kappa_{p}\left(\iota_{n-1}, \iota_{n}\right), \kappa_{p}\left(\iota_{n}, \iota_{n+1}\right)\right\}\right)^{k} .
\end{aligned}
$$

Thus

$$
1<\Theta\left(\kappa_{p}\left(\iota_{n}, \iota_{n+1}\right)\right) \leqslant \Theta\left(\max \left\{\kappa_{p}\left(\iota_{n-1}, \iota_{n}\right), \kappa_{p}\left(\iota_{n}, \iota_{n+1}\right)\right\}\right)^{k} .
$$

If there exists $n \in \mathbb{N}$ such that $\Theta\left(\max \left\{\kappa_{p}\left(\iota_{n-1}, \iota_{n}\right), \kappa_{p}\left(\iota_{n}, \iota_{n+1}\right)\right\}\right)^{k}=\kappa_{p}\left(\iota_{n}, \iota_{n+1}\right)$, then (2.3) becomes

$$
1<\Theta\left(\kappa_{p}\left(\iota_{n}, \iota_{n+1}\right)\right) \leqslant \Theta\left(\kappa_{p}\left(\iota_{n}, \iota_{n+1}\right)\right)^{k}<\Theta\left(\kappa_{p}\left(\iota_{n}, \iota_{n+1}\right)\right),
$$

which is a contradiction. Therefore $\Theta\left(\max \left\{\kappa_{p}\left(\iota_{n-1}, \iota_{n}\right), \kappa_{p}\left(\iota_{n}, \iota_{n+1}\right)\right\}\right)^{k}=\kappa_{p}\left(\iota_{n-1}, \iota_{n}\right)$, for all $n \in \mathbb{N}$. Thus from (2.3), we get

$$
\begin{aligned}
1 & <\Theta\left(\kappa_{p}\left(\iota_{n}, \iota_{n+1}\right)\right) \leqslant \Theta\left(\kappa_{p}\left(\iota_{n}, \iota_{n+1}\right)\right)^{k} \\
& \leqslant \Theta\left(\kappa_{p}\left(\iota_{n-1}, \iota_{n}\right)\right)^{k^{2}} \\
& \leqslant \Theta\left(\kappa_{p}\left(\iota_{n-2}, \iota_{n-1}\right)\right)^{k^{3}} \\
& \vdots \\
& \leqslant \Theta\left(\kappa_{p}\left(\iota_{0}, \iota_{1}\right)\right)^{k^{n}} .
\end{aligned}
$$

Thus by (2.3), we have

$$
1<\Theta\left(\kappa_{p}\left(\iota_{n}, \iota_{n+1}\right)\right) \leqslant \Theta\left(\kappa_{p}\left(\iota_{0}, \iota_{1}\right)\right)^{k^{n}} .
$$

Taking $\mathrm{n} \rightarrow \infty$ in (2.4), we get

$$
\lim _{n \rightarrow \infty} \Theta\left(\kappa_{p}\left(\iota_{n}, \iota_{n+1}\right)\right)=1 .
$$


By $\left(\Theta_{2}\right)$, we get

$$
\lim _{n \rightarrow \infty} K_{p}\left(\iota_{n}, \iota_{n+1}\right)=0
$$

By $\left(\Theta_{3}\right), \exists 0<\mathrm{h}<1$ and $\vartheta \in(0, \infty]$ such that

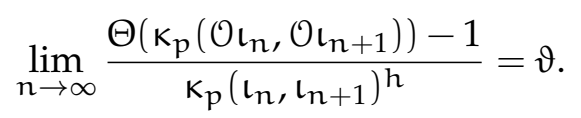

Assume that $\vartheta<\infty$. In this case, let $\lambda=\frac{\vartheta}{2}>0$. By definition, $\exists \mathrm{n}_{1} \in \mathbb{N}$ so that

$$
\left|\frac{\Theta\left(\kappa_{p}\left(\mathcal{O} \iota_{n}, \mathcal{O} \iota_{n+1}\right)\right)-1}{\kappa_{p}\left(\iota_{n}, \iota_{n+1}\right)^{h}}-\vartheta\right| \leqslant \lambda, \quad \forall n>n_{1} .
$$

This implies that

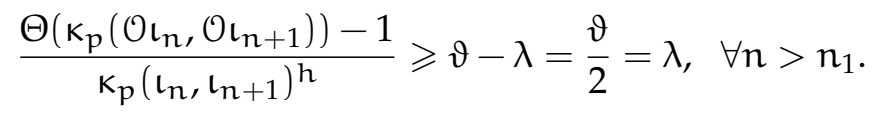

Then

$$
n \kappa_{p}\left(\iota_{n}, \iota_{n+1}\right)^{h} \leqslant \mu n\left[\Theta\left(\kappa_{p}\left(\mathcal{O} \iota_{n}, \mathcal{O} \iota_{n+1}\right)\right)-1\right], \quad \forall n>n_{1},
$$

with $\mu=\frac{1}{\lambda}$. Now we assume that $\vartheta=\infty$ and $\lambda>0$. By definition, $\exists n_{1} \in \mathbb{N}$ so that

$$
\lambda \leqslant \frac{\Theta\left(\kappa_{p}\left(\mathcal{O} \iota_{n}, \mathcal{O} \iota_{n+1}\right)\right)-1}{\kappa_{p}\left(\iota_{n}, \iota_{n+1}\right)^{h}}, \forall n>n_{1} .
$$

This implies that

$$
n \kappa_{p}\left(\iota_{n}, \iota_{n+1}\right)^{h} \leqslant \mu n\left[\Theta\left(\kappa_{p}\left(\mathcal{O} \iota_{n}, \mathcal{O} \iota_{n+1}\right)\right)-1\right], \quad \forall n>n_{1},
$$

where $\mu=\frac{1}{\lambda}$. Hence, in all situation, $\exists \mu>0$ and $n_{1} \in \mathbb{N}$ so that

$$
n \kappa_{p}\left(\iota_{n}, \iota_{n+1}\right)^{h} \leqslant \mu n\left[\Theta\left(\kappa_{p}\left(\mathcal{O} \iota_{n}, \mathcal{O} \iota_{n+1}\right)\right)-1\right]
$$

for all $n>n_{1}$. Thus by (2.4) and (2.5), we get

$$
n \kappa_{p}\left(\iota_{n}, \iota_{n+1}\right)^{h} \leqslant \mu n\left(\left[\left(\Theta\left(\kappa_{p}\left(\iota_{0}, \iota_{1}\right)\right)\right]^{k^{n}}-1\right) .\right.
$$

Taking $n \rightarrow \infty$, we obtain

$$
\lim _{n \rightarrow \infty} n \kappa_{p}\left(\iota_{n}, \iota_{n+1}\right)^{h}=0 .
$$

Hence, $\exists n_{2} \in \mathbb{N}$ so that

$$
\kappa_{p}\left(\iota_{n}, \iota_{n+1}\right) \leqslant \frac{1}{n^{1 / h}}, \quad \forall n>n_{2} .
$$

Consider the triangle inequality for $q \geqslant 1$, we have

$$
\begin{aligned}
\kappa_{p}\left(\iota_{n}, \iota_{n+q}\right) \leqslant & p\left(\iota_{n}, \iota_{n+1}\right) \kappa_{p}\left(\iota_{n}, \iota_{n+1}\right)+p\left(\iota_{n+1}, \iota_{n+q}\right) \kappa_{p}\left(\iota_{n+1}, \iota_{n+q}\right) \\
\leqslant & p\left(\iota_{n}, \iota_{n+1}\right) \kappa_{p}\left(\iota_{n}, \iota_{n+1}\right)+p\left(\iota_{n+1}, \iota_{n+q}\right) p\left(\iota_{n+1}, \iota_{n+2}\right) \kappa_{p}\left(\iota_{n+1}, \iota_{n+2}\right) \\
& +p\left(\iota_{n+1}, \iota_{n+q}\right) p\left(\iota_{n+2}, \iota_{n+q}\right) \kappa_{p}\left(\iota_{n+2}, \iota_{n+q}\right) \\
\leqslant & p\left(\iota_{n}, \iota_{n+1}\right) \kappa_{p}\left(\iota_{n}, \iota_{n+1}\right)+p\left(\iota_{n+1}, \iota_{n+q}\right) p\left(\iota_{n+1}, \iota_{n+2}\right) \kappa_{p}\left(\iota_{n+1}, \iota_{n+2}\right) \\
& +p\left(\iota_{n+1}, \iota_{n+q}\right) p\left(\iota_{n+2}, \iota_{n+q}\right) p\left(\iota_{n+2}, \iota_{n+3}\right) \kappa_{p}\left(\iota_{n+2}, \iota_{n+3}\right) \\
& +p\left(\iota_{n+1}, \iota_{n+q}\right) p\left(\iota_{n+2}, \iota_{n+q}\right) p\left(\iota_{n+3}, \iota_{n+q}\right) \kappa_{p}\left(\iota_{n+3}, \iota_{n+q}\right) \\
\vdots & \\
\leqslant & p\left(\iota_{n}, \iota_{n+1}\right) \kappa_{p}\left(\iota_{n}, \iota_{n+1}\right)+\sum_{i=n+1}^{n+q-2}\left(\prod_{j=n+1}^{i} p\left(\iota_{j}, \iota_{n+q}\right)\right) p\left(\iota_{i}, \iota_{i+1}\right) \kappa_{p}\left(\iota_{i}, \iota_{i+1}\right)
\end{aligned}
$$




$$
+\prod_{i=n+1}^{n+q-1} p\left(\iota_{i}, \iota_{n+q}\right) \kappa_{p}\left(\iota_{n+q-1}, \iota_{n+q}\right) .
$$

which further implies that

$$
\begin{aligned}
\kappa_{p}\left(\iota_{n}, \iota_{n+q}\right) \leqslant & p\left(\iota_{n}, \iota_{n+1}\right) \kappa_{p}\left(\iota_{n}, \iota_{n+1}\right)+\sum_{i=n+1}^{n+q-2}\left(\prod_{j=n+1}^{i} p\left(\iota_{j}, \iota_{n+q}\right)\right) p\left(\iota_{i}, \iota_{i+1}\right) \kappa_{p}\left(\iota_{i}, \iota_{i+1}\right) \\
& +\left(\prod_{i=n+1}^{n+q-1} p\left(\iota_{i}, \iota_{n}+q\right)\right) p\left(\iota_{n+q-1}, \iota_{n+q}\right) \kappa_{p}\left(\iota_{n}+q-1, \iota_{n+q}\right) \\
= & p\left(\iota_{n}, \iota_{n+1}\right) \kappa_{p}\left(\iota_{n}, \iota_{n+1}\right)+\sum_{i=n+1}^{n+q-1}\left(\prod_{j=n+1}^{i} p\left(\iota_{j}, \iota_{n+q}\right)\right) p\left(\iota_{i}, \iota_{i+1}\right) \kappa_{p}\left(\iota_{i}, \iota_{i+1}\right) \\
\leqslant & p\left(\iota_{n}, \iota_{n+1}\right) \kappa_{p}\left(\iota_{n}, \iota_{n+1}\right)+\sum_{i=n+1}^{n+q-1}\left(\prod_{j=0}^{i} p\left(\iota_{j}, \iota_{n+q}\right)\right) p\left(\iota_{i}, \iota_{i+1}\right) \kappa_{p}\left(\iota_{i}, \iota_{i+1}\right) \\
\leqslant & p\left(\iota_{n}, \iota_{n+1}\right) \kappa_{p}\left(\iota_{n}, \iota_{n+1}\right)+\sum_{i=n+1}^{n+q-1}\left(\prod_{j=0}^{i} p\left(\iota_{j}, \iota_{n+q}\right)\right) p\left(\iota_{i}, \iota_{i+1}\right) \frac{1}{i \frac{1}{k}} .
\end{aligned}
$$

Thus

$$
\kappa_{p}\left(\iota_{n}, \iota_{n+q}\right) \leqslant p\left(\iota_{n}, \iota_{n+1}\right) \kappa_{p}\left(\iota_{n}, \iota_{n+1}\right)+\sum_{i=n+1}^{n+q-1}\left(\prod_{j=0}^{i} p\left(\iota_{j}, \iota_{n+q}\right)\right) p\left(\iota_{i}, \iota_{i+1}\right) \frac{1}{i^{\frac{1}{k}}} .
$$

Now, consider

$$
\begin{aligned}
\sum_{i=n+1}^{n+q-1}\left(\prod_{j=0}^{i} p\left(\iota_{j}, \iota_{n+q}\right)\right) p\left(\iota_{i}, \iota_{i+1}\right) \frac{1}{n^{\frac{1}{k}}} & =\sum_{i=n+1}^{n+q-1} \frac{1}{i^{\frac{1}{k}}}\left(\prod_{j=0}^{i} p\left(\iota_{j}, \iota_{n+q}\right)\right) p\left(\iota_{i}, \iota_{i+1}\right) \\
& \leqslant \sum_{i=n+1}^{\infty} \frac{1}{i^{\frac{1}{k}}}\left(\prod_{j=0}^{i} p\left(\iota_{j}, \iota_{n+q}\right)\right) p\left(\iota_{i}, \iota_{i+1}\right)=\sum_{i=n+1}^{\infty} u_{i} v_{i},
\end{aligned}
$$

where

$$
\mathrm{u}_{\mathrm{i}}=\frac{1}{i^{\frac{1}{k}}}
$$

and

$$
V_{i}=\left(\prod_{j=0}^{i} p\left(\iota_{j}, \iota_{n+q}\right)\right) p\left(\iota_{i}, \iota_{i+1}\right)
$$

Since $\frac{1}{k}>0, \sum_{i=n+1}^{\infty} \frac{1}{i^{\frac{1}{k}}}$ converges and also $V_{i}=\left(\prod_{j=0}^{i} p\left(\mathfrak{l}_{j}, \mathfrak{l}_{n+q}\right)\right) p\left(\mathfrak{l}_{i}, \mathfrak{l}_{i+1}\right)$ is increasing and bounded above. Thus $\lim _{i \rightarrow \infty}\left\{V_{i}\right\}=\sup \left(V_{i}\right)$, exists and is non zero. Hence, the product $\left(\prod_{j=0}^{i} p\left(\iota_{j}, \iota_{n}+q\right)\right) p\left(\iota_{i}, \iota_{i+1}\right)$ converges. Thus $\sum_{i=n+1}^{\infty} u_{i} V_{i}$ converges. Let us consider the partial sum

$$
S_{q}=\sum_{i=0}^{q}\left(\prod_{j=0}^{i} p\left(\iota_{j}, \iota_{n+q}\right)\right) p\left(\iota_{i}, \iota_{i+1}\right) \frac{1}{i \frac{1}{k}}
$$


Now from (2.6), we have

$$
\kappa_{p}\left(\iota_{n}, \iota_{n+q}\right) \leqslant p\left(\iota_{n}, \iota_{n+1}\right) \kappa_{p}\left(\iota_{n}, \iota_{n+1}\right)+\left(S_{n+q-1}-S_{n}\right) .
$$

By ratio test and using the condition (2.2), we get guaranty of existence of $\lim _{n \rightarrow \infty} S_{n}$. Hence $\left\{S_{n}\right\}$ is Cauchy. Now taking $n \rightarrow+\infty$ in (2.7), we have

$$
\lim _{n \rightarrow \infty} K_{p}\left(l_{n}, l_{n+q}\right)=0,
$$

that is, $\left\{\iota_{n}\right\}$ is a Cauchy sequence in $\left(\mathcal{W}, \kappa_{p}\right)$, so $\left\{\iota_{n}\right\}$ converges to some $u \in \mathcal{W}$. Now we prove that $u=\mathcal{O} u$. Since $\mathfrak{l}_{n} \rightarrow \mathfrak{u}$ as $n \rightarrow \infty$ and the mapping $\mathcal{O}$ is continuous, so we have $\mathcal{O} \iota_{n} \rightarrow \mathcal{O} u$ as $n \rightarrow \infty$. Thus we have

$$
\kappa_{p}(u, \mathcal{O u})=\lim _{n \rightarrow \infty} \kappa_{p}\left(\iota_{n+1}, \mathcal{O u}\right) \lim _{n \rightarrow \infty} \kappa_{p}\left(\mathcal{O} \iota_{n}, \mathcal{O u}\right)=0,
$$

and hence $u=\mathcal{O} u$.

Theorem 2.3. Let $\left(\mathcal{W}, \mathrm{K}_{e}\right)$ be a complete Eb-MS and $\mathcal{O}: \mathcal{W} \rightarrow \mathcal{W}$. Assume that $\exists \mathrm{k} \in(0,1)$ and $\Theta \in \Omega$ such that

$$
\Theta\left(\kappa_{p}(\mathcal{O}, \mathcal{O} s)\right) \leqslant \Theta(M(\iota, s))^{k},
$$

where

$$
M(l, s)=\max \left\{\kappa_{p}(\iota, s), \kappa_{p}(\iota, \mathcal{O} \iota), \kappa_{p}(s, \mathcal{O} s), \frac{\kappa_{p}(\iota, \mathcal{O} \iota) \kappa_{p}(s, \mathcal{O} s)}{1+\kappa_{p}(\iota, s)}\right\}, \forall \iota, s \in \mathcal{W}
$$

with $\kappa_{p}(\mathcal{O} \iota, \mathcal{O} s)>0$. Suppose that these assertions also hold

(i) $\mathcal{O}$ is continuous;

(ii) $\sup _{m \geqslant 1} \lim _{i \rightarrow \infty} \frac{p\left(\mathfrak{\iota}_{i+1}, \iota_{i+2}\right) p\left(\mathfrak{\iota}_{i+1}, \iota_{m}\right)}{p\left(\mathfrak{\iota}_{i}, \iota_{i+1}\right)}<1$.

In addition, assume that, for every $\iota \in \mathcal{W}$, we have $\lim _{n \rightarrow \infty} p\left(\iota_{n}, \iota\right)$ and $\lim _{n \rightarrow \infty} p\left(\iota, \iota_{n}\right)$ exist and are finite. Then, $\exists \iota^{*} \in \mathcal{W}$ such that $\iota^{*}=\mathcal{O} \iota^{*}$.

Corollary 2.4. Let $\left(\mathcal{W}, \kappa_{e}\right)$ be a complete Eb-MS and $\mathcal{O}: \mathcal{W} \rightarrow \mathcal{W}$ be a rational type $(\alpha, \Theta)$-contraction such that:

(i) $\mathcal{O}$ is $\alpha$-admissible;

(ii) $\exists \iota_{0} \in \mathcal{W}$ such that $\alpha\left(\iota_{0}, \mathcal{O} \iota_{0}\right) \geqslant 1$;

(iii) $\mathcal{O}$ is continuous;

(iv) $\sup _{m \geqslant 1} \lim _{i \rightarrow \infty} \frac{p\left(\iota_{i+1}, \iota_{i+2}\right) p\left(\iota_{i+1}, \iota_{m}\right)}{p\left(\iota_{i}, \iota_{i+1}\right)}<1$.

In addition, assume that, for every $\iota \in \mathcal{W}$, we have $\lim _{n \rightarrow \infty} p\left(\iota_{n}, \iota\right)$ and $\lim _{n \rightarrow \infty} p\left(\iota, \iota_{n}\right)$ exist and are finite. Then, $\exists \iota^{*} \in \mathcal{W}$ such that $\iota^{*}=\mathcal{O} \iota^{*}$.

Proof. If we take $p(\mathrm{l}, z)=p(z, s)$ in above Theorem 2.2, we get the conclusion.

Corollary 2.5. Let $\left(\mathcal{W}, \mathrm{\kappa}_{\mathrm{b}}\right)$ be a complete $\mathrm{b}-\mathrm{MS}$ and $\mathcal{O}: \mathcal{W} \rightarrow \mathcal{W}$ be a rational type $(\alpha, \Theta)$-contraction such that:

(i) $\mathcal{O}$ is $\alpha$-admissible;

(ii) $\exists \mathfrak{\iota}_{0} \in \mathcal{W}$ such that $\alpha\left(\iota_{0}, \mathcal{O} \mathfrak{\iota}_{0}\right) \geqslant 1$;

(iii) $\mathcal{O}$ is continuous.

Then, $\exists \mathfrak{u} \in \mathcal{W}$ such that $\mathcal{O} u=u$.

Proof. If we take $\mathrm{p}(\mathrm{l}, z)=\mathrm{p}(z, \mathrm{~s})=\mathrm{b} \geqslant 1$ in above Theorem 2.2.

Corollary 2.6. Let $(\mathcal{W}, \mathrm{k})$ be a complete metric space and $\mathcal{O}: \mathcal{W} \rightarrow \mathcal{W}$ be a rational type $(\alpha, \Theta)$-contraction such that: 
(i) $\mathcal{O}$ is $\alpha$-admissible;

(ii) $\exists \mathfrak{\iota}_{0} \in \mathcal{W}$ such that $\alpha\left(\iota_{0}, \mathcal{O} \mathfrak{\iota}_{0}\right) \geqslant 1$;

(iii) $\mathcal{O}$ is continuous.

Then, $\exists \mathfrak{u} \in \mathcal{W}$ such that $\mathcal{O} u=u$.

Proof. If we take $p(l, z)=p(z, s)=1$ in above Theorem 2.2.

Example 2.7. Let $\mathcal{W}=\{0,1,2\}$. Define $p: \mathcal{W} \times \mathcal{W} \rightarrow[1, \infty)$ and $\kappa_{p}: \mathcal{W} \times \mathcal{W} \rightarrow[1, \infty)$ as $p(\iota, s)=1+\iota s$ and

$$
\begin{aligned}
& \kappa_{p}(2,2)=\kappa_{p}(0,0)=\kappa_{p}(1,1)=0, \\
& \kappa_{p}(2,0)=\kappa_{p}(0,2)=5, \kappa_{p}(1,0)=\kappa_{p}(0,1)=10, \\
& \kappa_{p}(1,2)=\kappa_{p}(2,1)=30 .
\end{aligned}
$$

Now, define

$$
\mathcal{O}: \mathcal{W} \rightarrow \mathcal{W}
$$

by

$$
\mathcal{O} \iota= \begin{cases}0, & \text { if } \iota \in\{0,2\} \\ 2, & \text { if } \iota=1\end{cases}
$$

and choose $k=\frac{3}{4}$. Define $\Theta(\beta)=e^{\sqrt{\beta}}$. Now we discuss various cases to prove the assumptions of our main result.

Case 01: If $\iota=0, s=1$, we have

$$
\begin{aligned}
\Theta\left(\kappa_{\mathfrak{p}}(\mathcal{O} 0, \mathcal{O} 1)\right)=\Theta\left(\kappa_{p}(0,2)\right) & =e^{\sqrt{5}}<\left(e^{\sqrt{10}}\right)^{\frac{3}{4}} \\
& =\left[\Theta\left(\max \left\{\kappa_{p}(0,1), \kappa_{p}(0, \mathcal{O} 0), \kappa_{p}(1, \mathcal{O} 1), \frac{\kappa_{p}(0, \mathcal{O} 0) \kappa_{p}(1, \mathcal{O} 1)}{1+\kappa_{p}(0,1)}\right\}\right)\right]^{\frac{3}{4}} .
\end{aligned}
$$

Case 02: If $\iota=0, s=2$, we have

$$
\begin{aligned}
\Theta\left(\kappa_{\mathfrak{p}}(\mathcal{O} 0, \mathcal{O} 2)\right)=\Theta\left(\kappa_{\mathfrak{p}}(0,0)\right) & =e^{0} \\
& <\left[\Theta\left(\max \left\{\kappa_{\mathfrak{p}}(0,2), \kappa_{\mathfrak{p}}(0, \mathcal{O} 0), \kappa_{\mathfrak{p}}(2, \mathcal{O} 2), \frac{\kappa_{\mathfrak{p}}(0, \mathcal{O} 0) \kappa_{\mathfrak{p}}(2, \mathcal{O} 2)}{1+\kappa_{\mathfrak{p}}(0,2)}\right\}\right)\right]^{\frac{3}{4}} .
\end{aligned}
$$

Case 03: If $\iota=1, s=2$, we have

$$
\begin{aligned}
\Theta\left(\kappa_{p}(\mathcal{O} 1, \mathcal{O} 2)\right) & =\Theta\left(\kappa_{p}(2,0)\right) \\
& =e^{\sqrt{5}}<\left(e^{\sqrt{30}}\right)^{\frac{3}{4}}<\left[\Theta\left(\max \left\{\kappa_{p}(1,2), \kappa_{p}(1, \mathcal{O} 1), \kappa_{p}(2, \mathcal{O} 2), \frac{\kappa_{p}(1, \mathcal{O} 1) \kappa_{p}(2, \mathcal{O} 2)}{1+\kappa_{p}(1,2)}\right\}\right)\right]^{\frac{3}{4}} .
\end{aligned}
$$

Case 04: If $\iota=s=0, \iota=s=1, \iota=s=2$, we have

$$
\begin{aligned}
\Theta\left(\kappa_{\mathfrak{p}}(\mathcal{O} \iota, \mathcal{O} s)\right) & =\Theta\left(\kappa_{\mathfrak{p}}(0,0)\right) \\
& =e^{0}<\left[\Theta\left(\max \left\{\kappa_{\mathfrak{p}}(\iota, s), \kappa_{\mathfrak{p}}(\iota, \mathcal{O} \iota), \kappa_{\mathfrak{p}}(s, \mathcal{O} s), \frac{\kappa_{p}(\iota, \mathcal{O} \iota) \kappa_{\mathfrak{p}}(s, \mathcal{O} s)}{1+\kappa_{\mathfrak{p}}(\iota, s)}\right\}\right)\right]^{\frac{3}{4}}, \forall s, \iota \in \mathcal{W} .
\end{aligned}
$$

Hence, all the conditions of above theorem are satisfied and $\mathcal{O}$ has a unique fixed point, which is, $\iota=0$.

We can establish variety of results as special cases of our main Theorem 2.2. 


\section{References}

[1] T. Abdeljawad, N. Mlaiki, H. Aydi, N. Souayah, Double Controlled Metric Type Spaces and Some Fixed Point Results, Mathematics, 6 (2018), 10 pages. 1

[2] J. Ahmad, A. S. Al-Rawashdeh, A. Azam, Fixed point results for $\{\alpha, \xi\}$-expansive locally contractive mappings, J. Inequal. Appl., 2014 (2014), 10 pages.

[3] J. Ahmad, A. Al-Rawashdeh, A. Azam, New fixed point theorems for generalized F-contractions in complete metric spaces, Fixed Point Theory Appl., 2015 (2015), 18 pages.

[4] B. Alqahtani, E. Karapinar, A. Öztürk, On $(\alpha-\psi)-K$-contractions in the extended b-metric space, Filomat, 32 (2018), 5337-5345.

[5] M. Arshad, A. Hussain, Fixed point results for generalized rational $\alpha$-Geraghty contraction, Miskolc Math. Notes, 18 (2017), 611-621.

[6] Z. Aslam, J. Ahmad, N. Sultana, New common fixed point theorems for cyclic compatible contractions, J. Math. Anal., 8 (2017), 1-12.

[7] A. Azam, N. Mehmood, J. Ahmad, S. Radenović, Multivalued fixed point theorems in cone b-metric spaces, J. Inequal. Appl., 2013 (2013), 9 pages.

[8] S. Banach, Sur les opérations dans les ensembles abstraits et leur application aux équations intégrales, Fund. Math., 3 (1922), 133-181.

[9] S. Czerwik, Contraction mappings in b-metric spaces, Acta Math. Inform. Univ. Ostraviensis, 1 (1993), 5-11. 1, 1.1

[10] H. P. Huang, S. Radenović, Some fixed point results of generalised Lipschitz mappings on cone b-metric spaces over Banach algebras, J. Comput. Anal. Appl., 20 (2016), 566-583.

[11] L.-G. Huang, X. Zhang, Cone metric spaces and fixed point theorems of contractive mappings, J. Math. Anal. Appl., 332 (2007), 1468-1476.

[12] N. Hussain, C. Vetro, F. Vetro, Fixed point results for $\alpha$-implicit contractions with application to integral equations, Nonlinear Anal. Model. Control, 21 (2016), 362-378.

[13] M. Jleli, B. Samet, A new generalization of the Banach contraction principle, J. Inequal. Appl., 2014 (2014), 8 pages. 1, $1.5,1$

[14] T. Kamran, M. Samreen, Q. UL Ain, A generalization of b-metric space and some fixed point theorems, Mathematics, 5 (2017), 7 pages. 1

[15] M. A. Kutbi, J. Ahmad, A. Azam, On fixed points of $\alpha-\psi$-contractive multi-valued mappings in cone metric spaces, Abst. Appl. Anal., 2013 (2013), 13 pages.

[16] N. Mlaiki, H. Aydi, N. Souayah, T. Abdeljawad, Controlled Metric Type Spaces and the Related Contraction Principle, Mathematics, 6 (2018), 7 pages. 1

[17] W. Onsod, T. Saleewong, J. Ahmad, A. E. Al-Mazrooei, P. Kumam, Fixed points of a $\Theta$-contraction on metric spaces with a graph, Commun. Nonlinear Anal., 2 (2016), 139-149.

[18] A. Shoaib, P. Kumam, A. Shahzad, S. Phiangsungnoen, Q. Mahmood, Fixed point results for fuzzy mappings in a b-metric space, Fixed Point Theory Appl., 2018 (2018), 12 pages.

[19] D. Wardowski, Fixed points of a new type of contractive mappings in complete metric spaces, Fixed Point Theory Appl., 2012 (2012), 6 pages. 1 